\title{
Sciences du jeu
}

$11 \mid 2019$

Que dit la philosophie des jeux vidéo?

\section{Gouverner le joueur dans les jeux vidéo}

La métaphysique des « affordances » au service de la politique des " architectures du choix »

Governing the Player in Video Games. From the Metaphysics of "Affordances" to the Politics of "Choice Architectures"

\section{Raphaël Verchère}

\section{(2) OpenEdition}

Journals

Édition électronique

URL : https://journals.openedition.org/sdj/1741

DOI : $10.4000 /$ sdj. 1741

ISSN : 2269-2657

\section{Éditeur}

Laboratoire EXPERICE - Centre de Recherche Interuniversitaire Expérience Ressources Culturelles

Education

\section{Référence électronique}

Raphaël Verchère, «Gouverner le joueur dans les jeux vidéo », Sciences du jeu [En ligne], 11 | 2019, mis en ligne le 16 avril 2019, consulté le 12 décembre 2022. URL : http://journals.openedition.org/sdj/1741 ; DOI : https://doi.org/10.4000/sdj.1741

Ce document a été généré automatiquement le 12 décembre 2022.

\section{(c)}

Creative Commons - Attribution - Pas d'Utilisation Commerciale - Pas de Modification 4.0 International - CC BY-NC-ND 4.0

https://creativecommons.org/licenses/by-nc-nd/4.0/ 


\section{Gouverner le joueur dans les jeux vidéo}

La métaphysique des « affordances » au service de la politique des

" architectures du choix »

Governing the Player in Video Games. From the Metaphysics of "Affordances" to

the Politics of "Choice Architectures"

Raphaël Verchère

1 Dans son usage contemporain, une affordance peut être définie comme un élément de l'environnement d'emblée signifiant, qui indique par sa seule présence l'usage qui peut en être fait : si elle est bien conçue, une poignée de porte suffit ainsi généralement à indiquer qu'elle en est une, et qu'elle sert à ouvrir la porte à laquelle elle est rattachée. En langue française, il est d'usage de conserver le terme affordance en anglais, les premiers psychologues français à l'utiliser l'ayant jugé intraduisible, bien que certains le rendent tout de même par "potentialité ». To afford peut signifier autant "offrir quelque chose", que "être capable de faire quelque chose", ou encore "être disponible » (Luyat et Regia-Corte, 2009, p. 299).

2 Les univers vidéoludiques bien constitués sont supposés enjoindre le joueur à l'action par leur seule constitution, et le concept d'affordance y occupe depuis quelque temps une place centrale pour cette raison. Les éléments qui les constituent doivent «afforder » naturellement des actions au joueur. Telle plateforme doit indiquer au joueur que son avatar peut marcher dessus; tel objet doit indiquer une menace et tel autre une récompense; tel autre objet ne doit au contraire rien "afforder ", et être délaissé.

Le concept d'affordance s'est par conséquent répandu dans la théorie contemporaine du design, et, par extension, dans celle du design de jeu. Cependant, malgré son usage, son emploi reste encore sous-théorisé par les professionnels du jeu vidéo. Formé à la fin des années 1970 par Gibson dans le champ de la psychologie de la perception, le concept d'affordance fut réinterprété avec une grande fortune par Norman dans le champ du design dans les années 1990, et c'est davantage cette interprétation qui est réinvestie 
par le design de jeu contemporain. Mais, ce faisant, toute la dimension ontologique et métaphysique associée à ce concept par Gibson, qui l'inscrivait dans une nouvelle théorie psychologique de la perception entendant dépasser la compréhension classique de la vision, est négligée. L'affordance se trouve réduite à un simple outil de design de jeu décontextualisé de sa portée originale, usage certes très fécond, mais qui n'en explore pas encore toutes les possibilités en termes d'agencement de la perception du joueur dans les jeux vidéo. Car, comme l'avaient déjà remarqué Joanna McGrenere et Wayne Ho, c'est d'abord par le travail de Norman que le concept d'affordance a été intégré aux études des Interfaces Homme-Machine (IHM), et a fortiori dans les études vidéo-ludiques (McGrenere et Ho, 2000, p. 1), mais ceci dans une relative ignorance de sa première conceptualisation par Gibson.

4 Dans une perspective d'épistémologie historique, l'objet de cet article est tout d'abord de dresser une généalogie de ce concept, de Gibson à Norman, pour en montrer les enjeux, et en souligner la pertinence de l'emploi dans le domaine du jeu vidéo, en en indiquant les différents usages possibles. Mais dans le même temps, il est aussi d'exposer le glissement et l'appauvrissement métaphysique du concept d'affordance, conduisant de Gibson à Norman. Il sera ici mis en évidence que certaines potentialités perceptives de ce concept restent encore peu explorées par le design de jeu contemporain, qui entend les affordances davantage dans le sens appauvri de Norman, que dans celui métaphysique et ontologique de Gibson. Enfin, il s'agira aussi de décrire la façon dont les affordances dans les jeux vidéo s'inscrivent dans une philosophie politique sous-jacente à la manière de gérer la liberté laissée au joueur dans sa pratique.

5 L'intérêt d'une approche philosophique est ici de se placer à l'intersection des différents savoirs produits jusqu'à présent sur l'emploi des affordances par les jeux vidéo, afin de les penser d'une manière systématique. L'ambition de cet article est ainsi d'analyser la manière dont le design de jeu entrecroise implicitement sur ce sujet des affordances une question de philosophie de la perception, qui intéresse la psychologie, avec une autre question qui relève de la philosophie politique, qui intéresse les sciences politiques. Au final, il s'agit de montrer ce qu'une notion d'affordance enrichie, aussi bien par le retour à l'écologie de la perception de Gibson que par sa réinterprétation en termes de philosophie politique, pourrait apporter à la conceptualisation des expériences de jeu.

\section{Généalogie des affordances}

\section{Les affordances dans la théorie du design de jeu contemporain}

6 Le concept d'affordance interroge les professionnels du jeu vidéo, qui tentent de le théoriser, mais sans souvent en épuiser toutes les potentialités. Un examen des espaces d'échange des savoirs professionnels montre que ce concept est régulièrement mobilisé, mais sans nécessairement faire droit à toute l'extension de ses enjeux théoriques. Sur Gamasutra, près de deux mille articles y font référence, mais un seul, signé Ara Shiriniran, évoque conjointement l'héritage de Gibson et de Norman, tout en éludant toute réflexion d'ensemble sur le sens de ce concept (Shirinian, 2012). L'ouvrage fondateur de Katie Salen et de Eric Zimmerman publié en 2003, Rules of Play: Game Design Fundamentals, n'emploie que par trois fois le terme, mais sans prendre le 
soin de le définir, comme si son sens allait de soi (Salen et Zimmerman, 2003, p. 537-538, 548). L'ouvrage tout aussi important de Jesse Schell publié en 2008, The Art of Game Design, n'y fait, quant à lui, même pas allusion (Schell, 2008). Quant aux différents auteurs de la double bible The Video Game Theory Reader (Wolf et Perron, 2003) et The Video Game Theory Reader 2 (Perron et Wolf, 2009), quelques-uns d'entre eux seulement mobilisent le terme, mais sans vraiment chercher à le conceptualiser (ce qui ne peut être reproché aux différents auteurs, compte tenu de l'objectif de l'ouvrage) exception faite d'Andreas Gregerson et de Torben Grodal (Grodal, 2003, p. 147-152; Gregersen et Grodal, 2009, p. 69), qui en font un usage pertinent pour montrer en quoi la perception détermine l'action dans les jeux vidéo, mais en le décontextualisant grandement du sens qu'en avait donné Norman, et surtout Gibson. L'espace «Game Development» de StackExchange fait ressortir essentiellement une seule discussion, interrogeant d'une façon générale ce «que sont les "affordances" dans les jeux vidéo » (Auteur anonyme [Evorlor], 2016).

7 De même, des formations au design de jeu proposent régulièrement des modules permettant d'appréhender son usage, tant de façon théorique que pratique. Parmi de nombreux exemples, citons sur Elephorm, un site de formation en ligne, le cours intitulé "Les notions d'ambiguïté et d'affordance dans le Game Design (Planques, 2014)», ou encore la formation dispensée au sein du cnam-enjmin (L'École nationale du jeu et des médias interactifs numériques, créée au sein du Conservatoire national des arts et métiers). Le "Collectif BAM", qui se présente lui-même comme "un groupe de designers engagés sur les pratiques numériques et collaboratives", a pour sa part organisé en 2016 une journée d'étude intitulée «affordance et jeux vidéo » (Thibault, 2016).

\section{Naissance des affordances : l'approche écologique de la perception visuelle de Gibson}

8 Le concept d'affordance naît à proprement parler durant les années 1970, dans les écrits du psychologue américain James J. Gibson. Gibson donne un premier article en 1977 intitule The Theory of affordances, repris ensuite en 1979 dans un livre inscrivant ce concept dans une théorie de la perception plus complète intitulée The Ecological Approach to Visual Perception (Gibson, 1986). Il fut quelques fois débattu de déterminer quelle était l'originalité de Gibson par rapport à d'autres théories de la perception antécédentes. La psychologie de la forme, l'approche phénoménologique de MerleauPonty, le pragmatisme de William James, ou même certaines théories de Bergson, paraissent mettre en avant des idées approchantes, ce que ne dénie pas Gibson luimême, concernant par exemple les théories de la Gestalt (Gibson, 1986, p. xiii). Mais, selon ses propres mots, Gibson donne une autre extension à ces idées, son concept d'affordance étant doté d'une singularité irréductible à d'autres théories.

9 Selon Gibson, « les affordances de l'environnement sont ce qu'il offre à l'animal, ce qu'il lui fournit ou lui met à disposition, pour le meilleur ou pour le pire» (Gibson, 1986, p. 127). L'environnement est donc directement signifiant, contrairement à ce que pourrait soutenir une conception naïve de la perception. Selon Gibson, d'ordinaire, la perception est - faussement - décrite comme fonctionnant suivant les étapes suivantes (Gibson, 1986, p. 1) :

1. L'œil fonctionne comme une caméra ou un appareil photo, qui enregistre des images. 
2. Les images sont ensuite transmises au cerveau.

3. L'esprit - supposé loger dans le cerveau - analyse ces images et produit du sens, de la signification, des valeurs.

Contre cette conception que l'on pourrait appeler « cognitiviste », Gibson oppose une approche « écologique » soutenant que la perception est d'emblée signifiante pour la pratique. Les objets nous informent de leurs usages possibles au moment même où nous les percevons.

Si une surface terrestre est presque horizontale (plutôt qu'inclinée), presque plate (plutôt que convexe ou concave), suffisamment étendue (relativement à la taille de l'animal), et que sa substance est rigide (relativement au poids de l'animal), alors la surface afforde le support. Il s'agit d'une surface de support, et on l'appelle couche, terre ou sol. Elle est se-tenir-dessus-able [stand-on-able], permettant une posture debout pour les quadrupèdes et les bipèdes. Elle est par conséquent marchable [walk-on-able] et courable [run-over-able]. (Gibson, 1986, p. 127)

11 Si la perception est signifiante pour la pratique, c'est parce qu'elle est indissociable de l'action. La vision est active, et, hormis dans les conditions artificielles de la psychologie de laboratoire où des dispositifs d'immobilisation sont parfois utilisés, l'œil n'est jamais fixe et stable tel une caméra. Au contraire, "lorsque aucune contrainte n'est mise sur le système visuel, nous regardons autour, nous nous levons vers quelque chose d'intéressant et nous tournons autour pour regarder la chose sous tous les côtés, et passons d'une perspective à une autre. Ceci est la vision naturelle » (Gibson, 1986, p. 1). Ce sont ces différents mouvements que nous faisons à plusieurs niveaux qui nous permettent de construire notre perception de la réalité. D'où la distinction de quatre niveaux de vision par Gibson :

-Vision photographique («snapshot vision ») : fixation d'un point d'une manière immobile par l'œil.

-Vision oculaire (« aperture vision ») : analyse d'un objet par les mouvements de l'œil, comme à travers un objectif ou un trou de serrure.

-Vision ambiante (" ambient vision ») : ce qui est perçu grâce aux mouvements de la tête.

-Vision ambulatoire ("ambulatory vision ») : ce qui est perçu grâce aux mouvements du corps dans ses déplacements.

12 Ces différents mouvements sont perçus par le corps au travers de la proprioception, et la perception du monde extérieur, c'est-à-dire l'exteroception, est indissociable de cette proprioception. La perception du monde ne peut être construite que sur le fondement d'une perception globale du moi, que Gibson nomme "egoreception »: «l'egoreception accompagne l'exteroception, comme l'autre côté d'une pièce de monnaie » (Gibson, 1986, p. 126). Cette étroite intrication de la perception du monde extérieur et des états internes conduit Gibson à remettre en cause les dualismes tant du corps et de l'esprit que du sujet et de l'objet.

Percevoir le monde est se copercevoir. Ceci est totalement en contradiction avec les dualismes de n'importe quelle forme, qu'il s'agisse du dualisme esprit-matière ou du dualisme esprit-corps. La conscience du monde et des relations complémentaires de soi au monde ne sont pas séparables (Gibson, 1986, p. 141).

\section{L'écologie de la perception en jeux vidéo}

Dans quelle mesure le jeu vidéo opère-t-il un pareil dépassement du dualisme ? Comme le souligne Mathieu Triclot: 
le jeu n'est ni un phénomène du moi, du psychisme à l'état pur, ni un phénomène qui relèverait simplement de l'extériorité, du dehors, du non-moi. L'espace du jeu s'instaure dans une forme de relation magique entre le sujet et l'extériorité, avec des objets extérieurs qui sont en quelque sorte habités, envahis par la subjectivité. Le réel du jeu n'est plus le réel de la perception ordinaire, celui de la complaisance soumise à une réalité extérieure à laquelle il faut s'ajuster et s'adapter (Triclot, 2011, p. 25-26).

En fait, la perception ordinaire comprise par Gibson se situe déjà dans un pareil entredeux. Peut-être le jeu vidéo en donnerait comme la représentation en acte. Pour paraphraser Foucault décrivant Les Ménines de Vélasquez, le jeu vidéo constituerait comme «la représentation de la représentation» (Foucault, 2010, p.31), non plus classique, mais écologique. C'est sans doute ce qui donne à l'expérience vidéoludique une importance métaphysique fondamentale: une sorte d'expérimentation du travail de réflexivité du sujet à l'endroit du réel, qui s'y autosaisit dans une sorte de tautologie idéaliste.

On pourrait objecter que les jeux vidéo en sont souvent restés à une conception cognitiviste de la perception visuelle. Les jeux à la première personne semble être englués dans une vision photographique («snapshot vision») relativement statique. Comme le remarque Mathieu Triclot :

si la vue $f p s$ imite en un sens la vision naturelle en adoptant un champ de vision plus restreint que celui que nous donnerait un plan large, elle oublie en même temps que la vision naturelle n'est jamais fixe, mais que le cerveau biologique reconstitue un sentiment de l'espace à coups de microregards permanents dans toutes les directions du champ visuel. La vision est stable, mais l'œil constamment en mouvement. C'est précisément ce mouvement continu qui disparaît de la vue fps, une absence qui nous cantonne à une perspective unique, soit celle de la proie à qui manque la perception de l'espace alentour, soit celle du chasseur à l'attention entièrement absorbée par sa cible, un mode de vision qui redouble celui du joueur focalisé sur l'écran et qui ne peut plus faire usage de la vision périphérique (Triclot, 2011, p. 83).

16 Cependant, ce serait oublier que la vue à la première personne, de par sa modalité (voir DOOM, id Software, 1993), est une «incitation à l'action» (Triclot, 2011, p. 86). La perception lacunaire pousse, du fait de son incomplétude, au mouvement. Finalement, l'action devient première dans le jeu vidéo, et pose d'emblée le joueur dans une vision écologique dépendante de l'action. Il serait alors possible de proposer une taxinomie des jeux vidéo à partir des quatre visions de Gibson. Par exemple, si les jeux de tir à la première personne sont fondés sur des visions ambulatoire (déplacement de l'avatar) et ambiante (rotation de l'avatar), il semble que cette dernière modalité soit incomplète dans la plupart des jeux en vue à la troisième personne où les mouvements de rotation ne font pas découvrir pleinement l'environnement.

17 Cet entremêlement de l'action et de la perception dans les jeux vidéo trouve son plein aboutissement dans les réalisations vidéoludiques accordant une grande importance à la lumière. Toute la théorie écologique de la perception visuelle repose en effet chez Gibson sur son concept de «lumière ambiante ». Le stimulus visuel est bien plus qu'un simple assemblage de points lumineux : il est déjà une image pleinement informée. La lumière est chargée d'informations, qui illumine (optique écologique) le sujet plus qu'elle ne l'irradie (optique physique) (Gibson, 1986, p. 47). Les différents mouvements réalisés par le sujet influent sur la lumière perçue, qui, de ce fait, informe sur la nature de l'environnement au même moment où elle illumine le sujet. Cette lumière ambiante 
fonde les «configurations optiques ambiantes» (Gibson, 1986, p. 65) perçues par le sujet, qui permettent notamment la saisie des surfaces composant les objets, et fondant dans le même temps les affordances correspondantes. Les jeux vidéo parviennent encore rarement à fonder les affordances par la seule perception d'une lumière ambiante changeante, sans doute parce que la plupart des moteurs de rendu, comme Unity, sont construits selon des théories optiques physiques et non encore écologiques. Un moteur de rendu parfaitement gibsonien est encore à créer.

On voit que Gibson s'est surtout intéressé à la perception visuelle. Il serait possible de donner une portée plus générale au concept d'affordance, en parlant d'affordance auditive, olfactive, tactile, gustative, ou même d'affordance perceptive en général. Toutefois, dans le cadre d'une étude du jeu vidéo, il paraît justifié de se restreindre uniquement au concept d'affordance visuelle. Les jeux vidéo sont, précisément, vidéo, et s'adressent ainsi dans leur projet même, pour ne pas dire dans leur essence, principalement à la vision du joueur, comme en témoigne le terme dans la plupart des langues. Bien qu'ils sollicitent souvent d'autres sens que la seule vision, la plupart des jeux vidéo n'en font qu'un usage accessoire ou redondant. Accessoire, car il est généralement possible de s'en passer : jouer à Grand Theft Auto (Rockstar, 1997-2013) en coupant le son ou sans manette vibrante est réalisable, alors que cela relève généralement de l'exploit avec un écran éteint. Redondant, car lorsque le son ne se réduit pas à une musique, il se contente souvent d'afforder une information déjà présente dans le champ visuel : tel bruit de détonation pour une grenade explosant dans la franchise Call of Duty (Activision, 2003-2018).

L'exploration d'affordances qui pourraient, par exemple, n'être qu'auditives, ne demeure pour l'instant que l'apanage de productions vidéoludiques plutôt restreintes, comme certains jeux vidéo destinés aux déficients visuels (Gaudy, 2010) - comme A Blind Legend (Dowino, 2015) -, qui peinent à se démocratiser. L'étude très complète de Karen Collins sur l'utilisation du son dans les jeux vidéo ne mobilise pas l'appareil conceptuel des affordances, qu'il s'agisse de celui de Gibson ou de Norman. Dans son examen des "fonctions de jeu audio", Collins insiste surtout sur l'idée que le son, lorsqu'il est utilisé sous la forme de symboles sonores ou de leitmotivs, est là " pour aider le joueur dans l'identification d'autres personnages, états d'esprit, environnements et objets, pour aider le jeu à devenir plus compréhensible et pour diminuer le temps d'apprentissage pour les nouveaux joueurs» (Collins, 2008, p. 130). Autrement dit, il amplifie le sens supposé être signifié d'emblée par la perception visuelle, comme s'il ne venait que s'y surajouter. Il y aurait pourtant ici une voie à explorer plus avant, l'idée d'affordance auditive dans les jeux vidéo étant parfaitement pertinente, comme l'avait déjà remarqué Guillaume Roux-Girard (2009, p. 95-101).

Ce traitement asymétrique et hiérarchisé des différentes perceptions est à ce point patent que les concepteurs cherchent généralement le moyen d'afforder visuellement ce qu'affordent les autres sens. Ainsi, pour reprendre l'exemple de la grenade, les Call of Duty font clignoter une grenade sur l'écran, pour redonder l'affordance auditive de la chute de celle-ci à proximité du joueur, au point de pouvoir se substituer même au sens de l'audition, et de rendre cette dernière dispensable. Le traitement d'autres sensations est parfois encore plus complexe, comme celui des odeurs, les technologies mises en œuvre dans les dispositifs de jeu n'étant pas encore parvenus à reproduire des stimuli de cet ordre de manière convaincante. Divers palliatifs sont employés: montrer visuellement l'odeur (moyen simple et direct), ou bien, plus complexe, faire tousser 
l'avatar lors de sa perception d'une odeur (de l'odeur au son), puis faire se secouer l'avatar lors de sa toux (du son au visuel).

\section{De la psychologie au design de jeu, ou les affordances selon Norman}

21 Le concept d'affordance proposé par Gibson est ensuite sorti de son cadre purement psychologique lorsqu'il fut popularisé par Donald Norman dans les années 1980 dans le domaine du design, dans son ouvrage The Design of Everyday Things ${ }^{1}$ en 1990. Norman (1990) détache ce concept de tout le cadre écologique dans lequel Gibson l'avait inscrit. Gibson avait pensé une théorie globale de la perception, s'opposant aux approches behavioriste et cognitiviste, qui entendait dépasser le dualisme du corps et de l'esprit. Ces enjeux métaphysiques sont évacués dans l'approche de Norman, qui ne s'intéresse qu'aux aspects purement fonctionnels de l'affordance.

Notamment, Norman inscrit les affordances dans un cadre très cognitiviste, à rebours de ce que proposait Gibson. Il y a selon lui :

sept étapes dans l'action: une pour les buts, trois pour l'exécution, et trois pour

l'évaluation:

- Former un but.

- Former une intention.

- Spécifier une action.

- Exécuter l'action.

- Percevoir l'état du monde.

- Interpréter l'état du monde.

- Évaluer le résultat (Norman, 1990, p. 48).

Dans son usage à destination du design, Norman redéfinit l'affordance comme suit :

le terme affordance fait référence aux propriétés perçues et réelles de la chose, essentiellement à ces propriétés fondamentales qui déterminent tout simplement comment une chose peut potentiellement être utilisée. Une chaise afforde ("est faite pour") le soutien, et, par conséquent, afforde l'asseoir (Norman, 1990, p. 9).

C'est avant tout ce concept normanien de l'affordance qui est employé dans le jeu vidéo, affordance dépouillée de tout ce que l'approche gibsonienne lui avait attaché dans son rapport au pragmatisme et au dépassement métaphysique du dualisme. L'affordance de Norman devient un simple outil, utilisable conjointement à d'autres pour concevoir des objets fonctionnels.

Les affordances sont précieuses pour les designers. Un objet correctement conçu permet d'imposer son usage conforme par sa seule perception. Nul besoin de mode d'emploi, de manuel, de notice, ni de tutoriel, rêve de tout concepteur. «Lorsque l'on tire parti des affordances, l'utilisateur sait quoi faire juste en regardant : aucun schéma, étiquette ou instruction n'est requis» (Norman, 1990, p. 9). La compréhension de l'usage d'une chose se fait d'une manière immédiate (à la fois sans délai et sans autre médiation entre le sujet et l'objet) et intuitive (au sens premier du terme : à partir de ce qui est vu). Cette leçon fut comprise par les jeux vidéo modernes, qui proposent de moins en moins de notices d'utilisation.

Les jeux vidéo - et les jeux en général - sont cependant un cas spécial de design. Dans le design d'objets classiques, les concepteurs doivent faire le maximum pour construire des affordances saisissables le plus facilement possible par les utilisateurs potentiels des objets. Un objet qui ne parvient pas correctement à afforder son usage dysfonctionne. 
Comme aime à le souligner Norman sur un mode très déculpabilisant pour les utilisateurs :

lorsque l'on a des problèmes avec des objets - que ce soit pour comprendre s'il faut pousser ou tirer une porte ou pour comprendre les caprices des ordinateurs modernes ou de l'industrie électronique - ce n'est pas notre faute. Ne vous blâmez pas vous-même: blâmez le designer. C'est la faute de la technologie, ou, plus précisément, du design (Norman, 1990, p. x).

Dans le design des objets ordinaires, quatre principes sont à respecter : offrir un modèle conceptuel sur le rôle que l'objet en question doit jouer dans le dispositif d'ensemble; fournir un feedback afin d'informer l'utilisateur des effets de son action; utiliser des contraintes afin d'interdire au maximum les mauvaises utilisations possibles d'un objet ; donner clairement des affordances perceptibles rendant claires les actions appropriées et dissimulant les mauvaises (Norman, 1990, p. xi-xii).

Avec le jeu vidéo en revanche, le design entre dans un domaine paradoxal où il s'agit de « rendre les tâches délibérément (et artificiellement) difficiles » (Norman, 1990, p. 124).

Les jeux, vidéo ou non, constituent une

catégorie dans laquelle les designers bafouent délibérément les lois de la bonne compréhension et bonne utilisation des objets. Les jeux sont supposés être difficiles. Et dans certains jeux, comme les jeux populaires d'aventure sur les ordinateurs de maison (ou de bureau) tels que Donjons et Dragons, tout l'enjeu est de deviner ce qui doit être fait, et comment (Norman, 1990, p. 205).

Mais si le design de jeu outrepasse ici les règles ordinaires du design - et encore ne le fait-il que de façon occasionnelle, la globalité du monde devant demeurer normalement signifiant dans son usage ordinaire -, il ne s'ensuit pas que le design de jeu ne possède aucune règle. En effet, «même les designs délibérément difficiles ne devraient pas être complètement difficiles» (Norman, 1990, p. 205): l'utilisateur doit éprouver des difficultés à réaliser la tâche au premier abord, mais la tâche doit tout de même être réalisable. Par ailleurs, les bonnes règles ordinaires du design doivent être gardées à l'esprit, afin de plus facilement aller à leur encontre, d'une manière toute paradoxale. Norman propose six «contre-règles ", qui peuvent s'illustrer fort bien dans les jeux vidéo (Norman, 1990, p. 206) :

- «Cacher les parties critiques, dissimuler les choses » : cas typique des jeux point-and-click, où il s'agit de découvrir progressivement à quoi peuvent servir les objets récoltés et autres éléments du décor.

- «Utiliser une cartographie qui ne soit pas naturelle pour l'exécution du cycle d'action, afin que la relation du contrôle aux choses qui doivent être contrôlées soit inappropriée ou hasardeuse »: rendre l'avatar ivre ou drogué comme dans Grand Theft Auto (Rockstar, 1997-2013) ou Batman (Warner Bros, 2009-2015), afin de rendre ses mouvements difficilement contrôlables.

- «Rendre les actions physiquement difficiles à réaliser » : telle combinaison de boutons sur Street Fighter II (Capcom, 1991), comme pour le célèbre «shoryuken » réclamant habileté et talent au gamepad (vers l'avant + vers le bas + diagonale vers le bas avant + coup de poing).

- «Imposer une manipulation physique précise dans un timing précis » : réaliser un lancer franc parfait au basket dans NBA 2K15 (2K Sports, 2014).

- «Ne pas donner de feedback du tout »: Super Mario Land (Nintendo, 1989), où le joueur, s'il ne se fie qu'à son expérience du jeu, est dans la plus complète ignorance sur combien de fois « Tatanga » doit être touché pour périr. 
- «Utiliser une cartographie qui ne soit pas naturelle pour l'évaluation du cycle d'action, afin que le système soit difficile à interpréter »: dans Indiana Jones and the Fate of Atlantis (LucasArt, 1992), lorsque Indiana doit manipuler des leviers afin de simuler un fantôme durant la conférence de Sophia, dont il est difficile de comprendre l'usage a priori. intentionnellement, cette liste a surtout valeur d'échantillon. Elle est certainement loin d'être exhaustive. Peut-être serait-il possible de réaliser une taxinomie des jeux vidéo à partir de la façon dont ils rendent les choses difficiles.

31

Norman peut être résumée comme suit (McGrenere et Ho, 2000, p. 3) :

- Pour Gibson, les affordances sont des possibilités d'action offertes par l'environnement, en relation avec les capacités d'action d'un sujet. Elles sont indépendantes de l'expérience du sujet, de sa connaissance ou de sa culture. Elles existent de façon binaire, au sens où elles existent, ou bien n'existent pas.

- Pour Norman, les affordances correspondent à des propriétés des objets qui peuvent ou non exister réellement. Elles sont des suggestions ou des indices sur comment utiliser un objet. Elles peuvent être dépendantes de l'expérience, de la connaissance ou de la culture du sujet. Elles peuvent rendre une action difficile ou au contraire la faciliter.

\section{Le difficile jeu politique de l'affordance}

\section{Gouverner par les affordances}

Tout l'enjeu du concepteur de jeu est alors de gouverner la conduite du joueur, presque dans un sens politique, en jouant sur sa perception des affordances. Notamment, en jouant sur l'adéquation entre l'objet et l'affordance qui en émane et qui peut en être perçue par le sujet. Car, comme l'explique Gaver, qui chercha à mettre les affordances au service du design d'interfaces de logiciels informatiques dans une application et un approfondissement des propositions de Norman :

les affordances en soi sont indépendantes de la perception. Elles existent, que le sujet percevant soit attentif à elles ou non, qu'elles soient perçues ou non, et même qu'il y ait pour elles des informations perceptives ou non. Par exemple, un verre d'eau afforde le fait de boire que j'ai soif ou non, une balle afforde le fait d'être jetée que quelqu'un le voit ou pas, et une crevasse afforde le fait de tomber même si elle est recouverte de broussailles. [...] Les exemples communs d'affordances font référence à des affordances perceptibles, dans lesquelles une information perceptive est disponible pour une affordance existante. Si aucune information perceptive n'est disponible pour une affordance existante, elle est cachée et doit être inférée à partir d'autres indices. Si les informations suggèrent une affordance qui n'existe pas, il y a une affordance trompeuse, sur laquelle des personnes pourraient essayer d'agir à tort. Enfin, les personnes ne penseront pas généralement à une action donnée lorsqu'il n'y a pas d'affordance pour elle, ni aucune information perceptive la suggérant (Gaver, 1991, p. 2).

D'où quatre grands types d'affordances à distinguer, l'usage d'un objet suggéré par sa perception subjective ne coïncidant pas nécessairement avec ce qu'il est effectivement objectivement possible d'en faire (Gaver, 1991, p. 2) : 


\begin{tabular}{lc|c|c|}
\cline { 3 - 3 } Information & Oui & Affordance trompeuse & Affordance perceptible \\
\cline { 3 - 4 } & Non & Rejet correct & Affordance cachée \\
\cline { 3 - 4 } & \multicolumn{2}{c}{ Non } & Oui
\end{tabular}

\section{Affordance} il n'est pas interdit de l'appliquer au cas des jeux vidéo, comme le font certains professionnels (Thibault, 2016). Ainsi, dans les jeux vidéo, un exemple parfait d'affordances perceptibles pourrait être la pièce dans Tetris (Nintendo, 1989) qui d'emblée donne sa signification : le carré livre immédiatement sa fonction qui est d'occuper un espace de $2 \times 2$ unités. Les affordances trompeuses pourraient quant à elles être constituées par tous les éléments des décors des jeux vidéo sur lesquels il est inutile d'agir: dans les GTA par exemple, les rues sont peuplées d'innombrables portes, qui toutes suggèrent qu'elles peuvent être ouvertes, mais qui pour la plupart demeurent closes. Mais ce sont sans doute les afforances cachées qui sont le cas le plus intéressant pour les jeux vidéo. Elles sont bien souvent attachées à des objets que les joueurs doivent utiliser pour progresser, mais dont le joueur ne comprend pas immédiatement l'usage : les jeux pointer-cliquer ont abondamment recours à ce dispositif, comme dans le jeu Day of the Tentacle (LucasArts, 1989), où le hamster n'afforde pas immédiatement ce à quoi il est destiné, à savoir, être mis dans un congélateur pour être cryogénisé, afin d'être ressuscité dans le futur après avoir été réchauffé dans un four à micro-ondes (avant cela en le revêtant d'un pull qui aura été mis dans une machine à laver pour le faire rétrécir), tout cela pour le faire courir dans une roue attachée à une dynamo produisant de l'électricité permettant de faire fonctionner une machine à remonter le temps.

Rendre les choses difficiles dans les jeux vidéo en utilisant ces affordances est une activité elle-même difficile. Norman prend l'exemple d'une adaptation vidéoludique de Donjons et Dragons (Gary Whisenhunt, Ray Wood, 1974), réalisée par ses étudiants :

Rendre les choses compliquées est une affaire délicate. Si le jeu n'est pas assez difficile, les joueurs expérimentés se désintéressent. Mais d'un autre côté, s'il est trop difficile, la joie initiale laisse la place à de la frustration. En fait, plusieurs facteurs psychologiques se jouent ici : le défi, le plaisir, la frustration, et la curiosité (Norman, 1990, p. 208).

36

montrent bien les fameuses vidéos du «Joueur du Grenier », constamment tiraillé entre l'ennui, lorsque le jeu est trop facile, et l'énervement, lorsqu'il est trop difficile. Norman signale quelques pistes afin de résoudre ce dilemme, vieilles recettes fréquemment employées par les concepteurs de jeu d'hier et d'aujourd'hui : «une approche est de saupoudrer sur le jeu de nombreux petits défis de difficultés variables. Une autre est d'avoir continuellement de nombreuses petites choses se produisant, afin de maintenir la curiosité éveillée » (Norman, 1990, p. 208). Toute cette mécanique de la difficulté, du rôle de l'échec, a évidemment été déjà grandement explorée par l'industrie du jeu vidéo et par ses théoriciens, comme Jesper Juul dans son livre The Art 
of Failure, qui montre en quoi les jeux vidéo sont fondés sur un "paradoxe de l'échec » que l'on peut résumer comme suit : «1) Nous évitons généralement d'échouer. 2) Nous faisons généralement l'expérience de l'échec lorsque nous jouons aux jeux. 3) Nous cherchons pourtant à jouer, bien que nous fassions l'expérience de quelque chose que nous évitons normalement» (Juul, 2016, p.2). Cependant, Juul n'aborde pas directement cette problématique par l'angle des affordances. Sans doute est-ce parce que cette logique de l'échec ne réside pas entièrement dans une question perceptive: elle tient aussi et avant tout à une notion de performance à réaliser dans un certain contexte, a fortiori lorsque l'environnement est correctement appréhendé par le joueur.

Concernant en revanche le seul niveau perceptif du jeu vidéo, les affordances devraient idéalement à elles seules suffire à résoudre cette difficulté inhérente au design de jeu. Être suffisamment perceptibles pour être utilisées de suite lorsqu'il le faut, être suffisamment dissimulées lorsqu'il est nécessaire de faire chercher le joueur. Mais, audelà des quatre types d'affordances reconnues par Gaver, il arrive parfois que les affordances proposées par les jeux soient insuffisantes. Une affordance insuffisante serait une affordance cachée, mais que le joueur ne parvient pas à découvrir par un fonctionnement classique du jeu. Sauf à renoncer, le joueur n'a que peu de choix pour progresser, qui se résument finalement à un seul : être informé, par un moyen ou par un autre, de ce qui doit être fait, de ce qui aurait dû être perçu, de ce qui a été perçu et aurait dû être utilisé. Cette stratégie peut revêtir des formes variées: demande de conseil à un joueur plus expérimenté, consultation des fameuses "soluces", utilisation de "cheat codes », contournement du fonctionnement usuel du jeu, etc.

Il est à remarquer que certains jeux ont directement implémenté dans leur jouabilité (gameplay) des fonctionnalités permettant de remédier aux affordances insuffisantes en rendant facilement visible l'invisible. Ainsi, les récents Batman proposent une vision en "mode détective ", tout comme les Assassin's Creed (Ubisoft, 2007-2018) proposent une «vision d'aigle». À l'aide de ces fonctionnalités, le joueur a accès à une autre perception de la réalité, autrement signifiante. Les affordances trompeuses sont généralement masquées (tel élément inutile du décor n'interfère plus dans la perception), et les affordances cachées sont montrées (tel élément du décor utilisable mais encore inaperçu est indiqué). D'autres fois, ce peut être un autre personnage du jeu qui indique explicitement ce que le joueur doit faire lorsque ce dernier a été bloqué trop longtemps.

Les concepteurs de jeu peuvent également agir sur une autre dimension de l'affordance lorsque le joueur ne parvient pas à les résoudre par le fonctionnement ordinaire du jeu. En effet, Gibson insiste sur le fait qu'une affordance est indissociable tant de la perception que de l'action. Vision d'aigle et mode détective agissent sur la perception; mais il est possible de rendre une affordance plus efficace en facilitant l'action qui doit en découler. La possibilité offerte par certains jeux de réassigner les contrôles de la manette de jeu (gamepad) pour des actions précises s'inscrit dans cette stratégie. Pour certains joueurs, l'action semblant la plus naturelle suite à une chose perçue peut être une réponse motrice particulière qui n'est pas celle attendue par les concepteurs $d u$ jeu. L'affordance induit une réponse qui n'est pas correcte : d'affordance perceptible, elle devient malencontreusement affordance trompeuse, et l'on pourrait fort bien parler ici d'une affordance ratée. Agir sur l'interface homme-machine en réassignant, comme le dirait Norman, une autre cartographie pour le cycle d'action, permet de corriger l'affordance. Pour les jeux vidéo, le grand classique de ces affordances ratées réside sans 
doute dans les jeux de simulation aérienne, où il s'agit de piloter l'un ou l'autre aéronef. Souvent, les concepteurs, très attachés à une sorte de réalisme et d'immersion, tiennent à ce que le gamepad ou le joystick simule stricto sensu le fameux «manche à balai » de l'aviation : l'incliner à gauche fait se tourner l'avion à gauche; l'incliner à droite le fait se tourner à droite... mais l'incliner vers le haut fait descendre l'avion, alors que l'incliner vers le bas le fait monter - comme pour un véritable avion. Malheureusement, pour de nombreux joueurs, surtout les débutants, mais également pour certains possédant déjà plusieurs heures de vol virtuel, cette association est totalement contre-intuitive. Lorsque cela est possible, ces joueurs préfèrent alors réassigner les contrôles, de façon à ce qu'appuyer sur le haut fasse véritablement voler vers le haut, et que le bas fasse voler vers le bas.

\section{L'affordance, solution du dilemme entre game et play?}

40 Ce problème de réassignation montre bien en quoi les affordances sont toujours relatives au sujet, leurs significations étant toujours dépendantes, comme le signalait Gaver, de la «culture, de l'expérience et de l'apprentissage» (Gaver, 1991, p. 3). Tel joueur habitué à jouer au football sur la franchise FIFA (Electronic Arts, 1993-2018) préférera souvent réassigner les contrôles de sa manette lorsqu'il jouera sur la franchise PES (Konami, 1995-2018), et vice versa. Il serait possible de réaliser une opération mentale permettant de s'adapter à cette autre culture du jeu sans réassignation, mais celle-ci représente toujours un coût (temps d'adaptation, effort, perte d'efficacité, etc.) que les joueurs sont rarement prêts à payer. Ce qui était parfaitement acquis et faisait figure d'automatisme sur un jeu redevient tâtonnant et demande tous les efforts de la conscience pour être réalisé sur un autre, créant une frustration qui est rarement le but du jeu - même si elle peut relever la figure d'un défi en certaines occasions. Cet exercice est similaire à celui réalisé par les musiciens devant «transposer à vue » une partition lorsqu'ils jouent, par exemple, d'un instrument transpositeur (comme une trompette en SiB devant jouer une partition d'orchestre). Peu y parviennent, et encore, la plupart ont recours à un stratagème consistant à imaginer une autre clé et d'autres altérations sur leur portée, ou encore pire, à utiliser un capodastre. Nombreux sont aussi ceux qui réécrivent l'ensemble de leur partition pour que les notes affordent des actions conformes à leur culture musicale d'origine.

Dans quelle mesure faut-il se fier au seul sujet dans son interprétation des affordances? Dans quelle mesure faut-il au contraire le guider? Comment laisser le joueur libre, tout en le contraignant? Il s'agit là de deux objectifs contradictoires, mais qui caractérisent les jeux dans leur essence. D'un côté, ils proposent une expérience de la liberté au joueur. Il y a précisément «jeu» (et sans doute plaisir), presque dans un sens mécanique, en ce que le joueur doit faire, et peut faire certains choix dans certaines situations: en ceci, le jeu vidéo s'écarte de l'expérience cinématographique, car le scénario joué n'est pas entièrement écrit d'avance. Mais d'un autre côté, les jeux vidéo proposent (généralement) un certain but à atteindre, qui contraignent dans le même temps cette liberté laissée au joueur : même si le joueur écrit un scénario, il en existe tout de même un a minima proposé par les concepteurs. La liberté laissée au joueur est donc encadrée dans certaines limites. L'expérience vidéoludique se distribue ainsi entre deux pôles. D'un côté, pour emprunter ces concepts à Roger Caillois (2006 [1958]), la pure paidia, où la pratique est très libre, rendant le jeu semblable à une sorte de jouet n'imposant a priori quasi aucun usage : peut-être Les Sims (Electronic Arts, 2000-2018) ou 
même MineCraft (Mojang, 2011) - voire Second Life (Linden Life, 2003), comme un cas limite. D'un autre côté, le pur ludus, où la pratique est contrainte par de nombreuses règles: Tetris (Nintendo, 1989), où, sauf à faire totalement fi des intentions des concepteurs, il est difficile de jouer sans observer strictement les règles. Comme le remarque Gonzalo Frasca, cette "distinction faite par Caillois entre paidia et ludus, décrit la différence entre "play" et "game" ( Frasca, 2003, p. 229, notre traduction), qui structure le design de jeu contemporain, le play et le game renvoyant, en schématisant, d'une part à l'expérience subjective du joueur, et d'autre part à la description objective de la logique interne du jeu (Triclot, 2011, p. 45, 61-67). Cette dimension subjective du jeu ne saurait être ignorée, ainsi que l'écrivait déjà Henriot :

qu'il s'agisse de jeu ou de jouet, ces réalités n'ont de sens et de fonction que parce qu'elles sont l'objet d'un joueur (play) qui tient lui-même au jeu que le joueur, par son attitude, introduit et maintient entre son jeu et lui. Jouant à (s'il s'agit d'un jeu), jouant avec (s'il s'agit d'un jouet), il se tient à distance. Le jeu, le jouet peuvent être définis d'une façon générale comme étant ce qui se prête au jeu. S'il y a jeu, le jeu n'est que dans l'attitude de l'acteur à l'égard de son acte (Henriot, 1969, p. 73).

Cette liberté dirigée, consistant à subtilement conduire cette expérience subjective (play) du joueur, a tout d'un oxymore. Les affordances permettent cependant de résoudre, en partie, ce dilemme entre la liberté du play et la contrainte du game antinomie que l'on retrouve de façon frappante dans le terme même de gameplay (jouabilité). Elles se situent dans un espace médian, entre subjectivité (liberté) et objectivité (contrainte). D'une part, une affordance dépend de la matérialité de l'objet. Une surface plane, comme une table dans notre monde quotidien, ou bien une plateforme dans un jeu vidéo, ne pourrait "afforder» la possibilité d'être "marchable ", si, par définition, elle n'était pas objectivement plane. En ce sens, sa matérialité objective impose une contrainte. Elle agit comme une sorte de «principe de réalité ", venant à la fois restreindre le champ des possibles du joueur, mais l'ouvrant également d'une manière symétrique : les tortues affordent dans les différents Mario à la fois une interdiction/impossibilité (marcher sur elles sans sauter dessus entraîne la mort) qu'une autorisation/possibilité (sauter sur elles permet de rebondir) - voire une obligation/nécessité dans certains cas (où il n'est pas possible de faire autrement pour progresser que de rebondir sur une tortue). Mais d'autre part, une affordance dépend du sujet qui est confronté à l'objet. Gibson, et même Norman, soulignent qu'une affordance est une relation entre le sujet et l'objet. Un objet n' "afforde " pas la même chose en fonction des sujets (et même, pour un sujet identique, il peut ne pas afforder de la même manière selon le contexte): dans un jeu vidéo, un joueur expérimenté ne comprend pas les objets de la même manière qu'un novice (le premier saura que la tortue est également portable et peut servir d'arme, alors que le second n'en comprendra qu'une menace), tout comme deux joueurs expérimentés peuvent ne pas les comprendre identiquement. Cet enracinement de l'affordance dans le sujet ouvre un espace de liberté, au sens où différents possibles, différentes virtualités se dessinent, inanticipables, imprévisibles a priori. Mais qui toutes ne seront pas fécondes, car corrigées par ce que le designer attend du joueur.

C'est ici que s'ouvrent des problèmes importants sur le(s) but(s) donné(s) à un jeu vidéo par ses créateurs. Il est difficile a priori d'être certain de la façon dont le régime d'affordances pensé par les concepteurs va véritablement être compris et utilisé par les joueurs. Il est rare qu'un jeu soit utilisé d'une manière parfaitement conforme au plan prévu. Des paradoxes surprenants peuvent apparaître, a fortiori avec la catégorie des 
jeux sérieux, genre de jeux vidéo les inscrivant sciemment dans une finalité d'apprentissage transcendant le seul plaisir du jeu. Comprises notamment selon Norman ou Gaver, les affordances dépendent par exemple de la culture, de l'expérience, du savoir du sujet. Les concepteurs des jeux sérieux organisent alors des affordances afin que le sujet, à terme, intègre cette culture, cette expérience, ce savoir attendu. Le succès dans le jeu n'est supposé être accordé par la jouabilité qu'à condition que les affordances soient comprises selon ces prérequis. Mais, comme le remarque très justement Sébastien Hock-Koon (2012, pp. 71-87), il est parfois possible que les joueurs jouent sans apprendre (avec « une affordance qui permet de jouer à un jeu vidéo sans en avoir appris les règles » : jouer à PONG (Atari Inc., 1972) sans pour autant intégrer les lois physiques du rebond), que les joueurs croient qu'ils aient compris (avec « une affordance permettant au joueur de croire qu'il a compris le jeu alors que ce n'est pas le cas » : penser que la réussite à Pac-Man (Namco, 1980) dépend du temps de réaction, alors qu'elle dépend en grande partie du fait de cerner le "caractère » des fantômes), ou encore qu'ils réussissent sans apprendre (avec «des affordances de réussite sans apprentissage »: l'emporter dans Civilization III (Infogrames, 2001) sans pour autant saisir les concepts historiques sous-jacents).

\section{Philosophie politique de la perception}

Ce problème qu'ont à résoudre les concepteurs de jeu consistant à concilier contrainte et liberté est finalement un problème de philosophie politique assez classique. Les affordances offrent - on oserait presque dire qu'elles « affordent » - une solution assez simple pour résoudre cette tension entre la contrainte et la liberté en agissant sur le seul niveau perceptif des sujets, en travaillant à partir de la conscience qu'ils ont du réel, en leur configurant un monde ayant pour ambition de les prédisposer à un certain type d'action. Cela n'a pas échappé à certains théoriciens contemporains du libéralisme, comme Thaler et Sunstein (2008), qui proposent, en partie à partir des affordances, une manière de gouverner paradoxale qu'ils nomment le "paternalisme libertarien ». Ce faisant, le dirigeant politique moderne théorisé selon cette vue se trouve dans une position analogue à celle du concepteur de jeu, et inversement. Organiser la société, organiser un jeu vidéo, consisterait en premier lieu à agir sur les représentations qui incitent, ou non, à l'action.

Par principe, les démocraties se refusent à agir d'une manière dirigiste et autoritaire vis-à-vis de leurs citoyens. Le libéralisme politique qui se théorise par exemple chez Kant craignait ce que ce dernier nommait le « paternalisme » :

un gouvernement qui serait fondé sur le principe de la bienveillance envers le peuple, comme celui d'un père envers ses enfants, c'est-à-dire un gouvernement paternaliste (imperium paternale), où les sujets sont forcés de se conduire d'une manière simplement passive, à la manière d'enfants mineurs, incapables de distinguer ce qui leur est vraiment utile ou nuisible (Kant, 1994, p. 65 / VIII, 291).

De fait, les jeux vidéo présentent souvent un mode "paternaliste » lorsque le joueur doit s'initier à son fonctionnement. Au contraire, la théorie de la démocratie moderne entend faire de la liberté remise à l'individu un principe absolu, point essentiel qui la fait s'opposer frontalement aux totalitarismes, qui radicalisent à l'excès l'idée de paternalisme, comme l'avait souligné par exemple Popper (1979, p. 85). Dans le cadre des démocraties libérales, la société devient-elle ingouvernable? Comment imprimer 
une direction à une société dont les acteurs, en définitive, sont caractérisés par une radicale liberté que l'on ne peut en aucun cas aliéner?

Le libéralisme ne fut pas avare de solutions, comme l'a montré Michel Foucault, en particulier dans son cours de 1979 intitulé Naissance de la biopolitique (Foucault, 2004) qui avait placé au centre de son étude la "gouvernementalité libérale ». L'«art de gouverner libéral », qui succède à la "raison d'État ", ne renonce pas à diriger la société. Il poursuit les mêmes objectifs, mais par des moyens différents, en apparence plus souples, capables d'agir malgré cette liberté inaliénable des sujets, en agissant sur leurs «conduites». Comme l'écrit Foucault dans "Le sujet et le pouvoir», «la "conduite" est à la fois l'action de "mener" les autres (selon des mécanismes de coercition plus ou moins stricts) et la manière de se comporter dans un champ plus ou moins ouvert de possibilités. L'exercice du pouvoir consiste à "conduire des conduites" et à aménager la probabilité » (Foucault, 2001, p. 1056). Gouverner est configurer un monde dans lequel des libertés ont à s'exercer. En façonnant correctement un monde, il est possible de remettre une pleine liberté aux sujets, qui ne pourront en faire d'autres utilisations que celles qui en sont normalement attendues.

Sans qu'ils aient visiblement connaissance de ces réflexions de Foucault, Richard $\mathrm{H}$. Thaler et Cass R. Sunstein ont fourni un contenu contemporain à cette question dans leur ouvrage Nudge paru en 2008, en proposant le concept de "paternalisme libertarien » («libertarian paternalism») (Thaler et Sunstein, 2008, p. 5). En apparence, cette expression a toutes les allures d'un oxymore. Thaler et Sunstein insistent pourtant sur ces deux aspects : libertarien, car « les gens devraient être libres de faire ce qu'ils préfèrent »; paternalisme, car «il est légitime d'influencer le comportement des gens afin de rendre leurs vies plus longues, plus saines, meilleures » (Thaler et Sunstein, 2008, p. 5). Libertarien intervient ici comme un qualificatif, afin d'euphémiser les côtés tranchants du paternalisme. La dimension paternaliste du gouvernement est tout à fait assumée par Thaler et Sunstein. Leur idée est simplement d'avoir un paternalisme préservant la liberté des individus, c'est-à-dire compatible avec les principes de la démocratie libérale.

Afin d'orienter les comportements des individus, Thaler et Sunstein proposent le concept d'« architecture du choix ». Comment orienter les comportements des gens ? Comment « conduire les conduites »? Le paternalisme usuel n'hésite pas à contraindre les individus. Le paternalisme libertarien se contente quant à lui d'inciter en configurant l'environnement, de sorte à ce que les individus prennent d'eux-mêmes les décisions attendues. «Un architecte du choix a la responsabilité d'organiser le contexte dans lequel les gens prennent leurs décisions » (Thaler et Sunstein, 2008, p. 3), et donc de l'organiser suffisamment habilement pour qu'un certain type de décision soit pris, plutôt qu'un autre. Principe en parfaite congruence avec la gouvernementalité décrite par Foucault :

le pouvoir ne s'exerce que sur des «sujets libres », et tant qu'ils sont «libres »entendons par là des sujets individuels ou collectifs qui ont devant eux un champ de possibilités où plusieurs conduites, plusieurs réactions et divers modes de comportement peuvent prendre place (Foucault, 2001, p. 1056).

Dans ce cadre, rien d'étonnant à ce que Thaler et Sunstein fassent référence dans leur ouvrage au " merveilleux livre de Don Norman, The Design of Everyday Things » (Thaler et Sunstein, 2008, p. 83). Le design et l'architecture du choix ont partie liée. Les éléments du design peuvent être une prise importante de pouvoir dans l'agir des 
individus. Ceci ouvre sur une philosophie politique du design, un simple objet pouvant cristalliser toute une conception politique $\mathrm{du}$ monde. Une simple affordance, correctement pensée, joue le rôle de "dispositif », au sens d'Agamben : «tout ce qui a, d'une manière ou d'une autre, la capacité de capturer, d'orienter, de déterminer, d'intercepter, de modeler, de contrôler et d'assurer les gestes, les conduites, les opinions et les discours des êtres vivants » (Agamben, 2007, p. 31).

51 Thaler et Sunstein suggèrent une politique dans laquelle des «nudges ", littéralement des " coups de coude ", servent d'éléments essentiels pour orienter les comportements - à la manière de ce parent éléphant qui, sur la couverture de leur livre, donne un coup de trompe à son petit pour l'inciter à avancer.

Un «coup de coude» [a nudge], ainsi que nous utiliserons l'expression, désigne n'importe quel aspect de l'architecture du choix qui altère le comportement des gens d'une façon prédictible sans interdire aucune option ou sans changer significativement leurs motivations économiques. Pour qu'une intervention compte comme un simple « coup de coude », elle doit être simple et non coûteuse à éviter. Les « coups de coude» ne sont pas des mandats. Placer des fruits à la hauteur des yeux compte comme un "coup de coude ». Bannir la « junk food» ne compte pas (Thaler et Sunstein, 2008, p. 6, notre traduction).

De fait, c'est bien ce que réalise le concepteur de jeu vis-à-vis de son joueur. Organiser la perception du joueur de façon à « altérer » son comportement et l'inciter à agir dans une certaine direction, mais sans pour autant supprimer ce qui ne doit pas ne pas être fait. Le concepteur d'un jeu doit "gouverner" ses joueurs, dans un sens foucaldien, c'est-à-dire «structurer le champ d'action éventuel » (Foucault, 2001, p. 1056), agencer des possibles, tel un " architecte du choix ", pour les inciter à aller dans une direction, sans pour autant les contraindre absolument.

Dans cette référence à l'architecture utilisée par Thaler et Sunstein, il y a plus qu'une simple métaphore. Un architecte, dans le sens usuel du terme, doit agencer l'espace afin de permettre à un bâtiment de fonctionner quant aux différentes personnes l'utilisant. Le concepteur de jeu en fait de même avec l'espace qu'il utilise, les univers ouverts du type GTA-like en étant l'expression la plus parfaite. Chose qui n'est pas simple à réaliser, en raison d'un double écueil. L'architecte du choix vidéoludique doit éviter de créer un simple "couloir " qui ôterait au joueur tout sentiment de liberté, mais aussi éviter d'enfanter un simple monde vierge de toute signification. En ce sens, le concepteur de jeu gouverne bien, puisque manipuler des espaces n'est rien d'autre que gouverner d'une façon libertaro-paternaliste. Il œuvre à la mise en place d'une « main invisible » devant guider son joueur à son insu.

Ce soubassement politique libéralo-paternaliste n'avait pas échappé aux concepteurs des premiers univers ouverts, bien qu'il ne fût encore saisi que d'une manière assez intuitive et implicite. Morningstar et Farmer furent les créateurs en 1985 du jeu Habitat, jeu célèbre pour avoir été l'un des premiers mondes virtuels en ligne massivement multijoueur, et pour se poser comme « délibérément ouvert " (Morningstar et Farmer, 1991, p. 287) afin de proposer aux joueurs une pluralité de buts différents à poursuivre. $\mathrm{Au}$ fur et à mesure de l'utilisation du jeu par les joueurs, Morningstar et Farmer comprirent vite qu'ils devraient moins se considérer comme des concepteurs de jeux, que comme "les directeurs d'une croisière durant un voyage sur l'océan ». Car fixant tout d'abord des objectifs précis aux joueurs (par exemple, une chasse au trésor), ils remarquaient aussitôt que "plus nous voulions impliquer les joueurs dans quelque chose, moins nous les contrôlions ». Il leur fallait changer d'approche, comme le 
montre ce constat: «nous pouvions influencer les choses, nous pouvions mettre en place des situations intéressantes, nous pouvions proposer des opportunités pour que les choses se produisent, mais nous ne pouvions pas prédire ni non plus dicter le résultat» (Morningstar et Farmer, 1991, p. 288). Ils durent alors devenir «des facilitateurs autant que des designers", pour enfin pouvoir disposer "d'une influence considérable sur le développement du système en dépit du fait que nous ne tenions pas le volant » (Morningstar et Farmer, 1991, p. 289). Sans doute n'est-ce pas un hasard si, dans leur réflexion, Morningstar et Farmer s'en remettent à des auteurs libéraux Popper, Hayek, Sowell - pour appuyer cette idée que « tout planisme détaillé centralisé est impossible » dans un jeu vidéo, et qu'il ne faut « même pas l'essayer » (Morningstar et Farmer, 1991, p. 285). Idée politique que tous les GTA-like postérieurs reprirent à leur compte - et peut-être même que toute jouabilité est forcé de suivre, celui-ci étant toujours recherche d'un équilibre entre contrainte du game et liberté du play. Se trouve ainsi peut-être cristallisé dans l'art du design de jeu la quintessence de ce nouvel art libéral de gouverner décrit par Foucault. Avec, comme outil principal, les affordances, qui se situent à l'intersection de la perception et de l'action.

\section{BIBLIOGRAPHIE}

AGAMBEN, G. (2007), Qu'est-ce qu'un dispositif ?, Paris, Rivages poche.

AUTEUR ANONYME [EVORLOR] (2016), « What are "affordances" in game design? - Game Development Stack Exchange », (consulté le 21 mars 2018).

CAILLOIS, R. (2006 [1958]), Les jeux et les hommes, Paris, Gallimard.

COLLINS, K. (2008), Game Sound - An Introduction to the History, Theory and Practice of Video Game Music and Sound Design, Cambridge, Mass, MIT Press.

FOUCAULT, M. (2001), « Le sujet et le pouvoir [1982] », dans Dits et Écrits II, Paris, Gallimard Quarto.

FOUCAULT, M. (2004 [1979]), Naissance de la biopolitique, Paris, Gallimard Seuil.

FOUCAULT, M. (2010 [1966]), Les Mots et les Choses, Paris, Gallimard

FRASCA, G. (2003), « Simulation versus Narrative : Introduction to Ludology », in M. J. P. Wolf et B. Perron (eds) The Video Game Theory Reader, New York ; London, Routledge, pp. 221- 233.

GAUDY, T. (2010), Conception de jeux vidéo sonores accessibles aux personnes aveugles, Saarbrücken, Univ Européenne.

GAVER, W. W. (1991), « Technology Affordances », dans Proceedings of the ACM CHI 91 Human Factors in Computing Systems Conference, New Orleans.

GIBSON, J. J. (1986 [1979]), The Ecological Approach to Visual Perception, New York, Taylor \& Francis. GREGERSEN, A. et GRODAL, T. (2009), « Embodiment and Interface », in B. Perron et M. J. P. Wolf (eds) The Video Game Theory Reader 2, New York, Routledge. 
GRODAL, T. (2003), « Stories for Eye, Ear, and Muscles : Video Games, Media, and Embodied Experiences ", dans The Video Game Theory Reader, New York ; London, Routledge.

HENRIOT, J. (1969), Le jeu, Paris, PUF.

HOCK-KOON, S. (2012), « Les affordances appliquées à l'absence d'apprentissage dans les jeux vidéo », Revue des Interactions Humaines Médiatisées, vol. 13, n 1, pp. 63-92

JUUL, J. (2016), The Art of Failure: An Essay on the Pain of Playing Video GamesReprint., Cambridge, Mass., MIT Press.

KANT, E. (1994), « Théorie et pratique », dans Théorie et pratique. D’un prétendu droit de mentir par humanité. La fin de toutes choses, Paris, GF Flammarion.

LUYAT, M., et REGIA-CORTE, T. (2009), «Les affordances : de James Jerome Gibson aux formalisations récentes du concept », L'Année psychologique, vol. vol. 109, n 2, pp. 297-332.

MCGRENERE, J. et HO W. (2000), « Affordances: Clarifying and Evolving a Concept », dans Proceedings of Graphics Interface 2000, Montreal, Canadian Human-Computer Communications Society

MORNINGSTAR, C., et FARMER F. R. (1991), « The Lessons of Lucasfilm's Habitat », in M. Benedikt (ed.) Cyberspace : First Steps, Cambridge, MIT Press, pp. 273-302

NORMAN, D. A. (1990), The Design of Everyday Things [1988], New York, Basic Books.

PERRON, B. et WOLF, M. J. P. (eds) (2009), The Video Game Theory Reader 2, New York, Routledge.

PLANQUES, T. (2014), Les notions d'ambiguité et d'affordance dans le Game Design, [en ligne],

« Elephorm », (consulté le 23 mars 2018).

POPPER, K. (1979 [1945]), La société ouverte et ses ennemis, tome 1 :l'ascendant de Platon, Paris, Seuil. ROUX-GIRARD, G. (2009), L'écoute de la peur : une étude du son dans les jeux vidéo d'horreur, Faculté des arts et des sciences, Université de Montréal.

SALEN, K. et ZIMMERMAN, E. (2003), Rules of Play: Game Design Fundamentals, Boston, MIT Press.

SCHELL, J. (2008), The Art of Game Design : A book of lenses, Amsterdam ; Boston, Morgan Kaufmann.

SHIRINIAN, A. (2012), « Intuition, Expectations and Culture: Learning from Psychology to Build Better Game Interfaces », (consulté le 15 novembre 2018).

THALER, R. H. et SUNSTEIN C. R. (2008), Nudge. Improving Decisions About Health, Wealth, and Hapiness, New Haven \& London, Yale University Press.

THIBAUlT, T. (2016), « Affordance \& jeux vidéo », dans Collectif Bam, (consulté le 23 mars 2018).

TRICLOT, M. (2011), Philosophie des jeux vidéo, Zones.

WOLF, M. J. P. et PERRON, B. (eds) (2003), The Video Game Theory Reader, New York, London, Routledge.

\section{NOTES}

1. Ouvrage déjà publié sous un autre titre, The Psychology of Everyday Things, en 1988. 


\section{RÉSUMÉS}

À la fin des années 1970, Gibson élabora le concept d' "affordance " pour désigner des objets qui apparaissent comme immédiatement signifiants dans le champ perceptif. Propre tout d'abord à la psychologie de la perception, ce concept s'est ensuite diffusé à d'autres domaines, notamment celui du design, en particulier au travers de l'ouvrage publié par le cogniticien Donald Norman en 1990, The Design of Everyday Things. Dans la conception des jeux vidéo, le concept d'affordance a une place toute trouvée, le design de jeu consistant généralement en l'élaboration d'un monde signifiant pour le joueur, qui doit idéalement l'appeler à l'action de par sa seule constitution et perception. De quelle manière les jeux vidéo organisent-ils et théorisent-ils la perception, d'une manière implicite ou explicite, par ce concept d'affordance? Son usage, parfois assumé, y est-il fécond et adapté ? À quelles limites ce concept est-il confronté ? Quelle est la philosophie politique sous-jacente à une telle manipulation du joueur à partir de ce qu'il perçoit?

In the late 1970s, Gibson built the concept of "affordance" to refer objects which are immediately significant in the perceptive area. First inherent to the psychology of perception, this concept has next been used in other fields, especially for design thanks to Donald Norman's book, The Design of Everyday Things, published in 1990. In video game conception, the affordance concept has a central place, because the game design is mainly about building a significant world for the player, which ideally must call to the action by its sole conception and perception. In which way video games do organize perception by this concept of affordance? Is its usage fruitful and fitted? What are the limits of this concept? What is the political philosophy which grounds this manipulation of the players based on what they perceive?

\section{INDEX}

Mots-clés : jeu vidéo, perception, affordances, nudging, design, Gibson, Norman, Gaver

Keywords : video game, perception, affordances, nudging, design, Gibson, Norman, Gaver

\section{AUTEUR}

\section{RAPHAËL VERCHÈRE}

Laboratoire sur les Vulnérabilités et les Innovations dans le Sport

Université Lyon 1 